\title{
Research article \\ A study of biochemical parameters in pregnant women in III trimester with non-alcoholic fatty liver disease (NAFLD)
}

\author{
Ganesh Pendli ${ }^{1}$, Chandana G. ${ }^{2}$, Surekha Balaji ${ }^{3}$, Devi Varaprasad M. ${ }^{4}$ \\ ${ }^{1}$ House Surgeon, ${ }^{3}$ Assistant Professor, Dept., of Radiology, ${ }^{4}$ Assistant Professor, Dept., of Community Medicine, PES Institute \\ of Medical Sciences and Research, Kuppam, 517425, Chittoor, Andhra Pradesh, India \\ ${ }^{2}$ Associate Professor, Dept., of Biochemistry, Kamineni Institute of Medical Sciences, Sreepuram Narketpally, 508254, \\ Nalgonda, Telangana, India
}

(Received: March $2021 \quad$ Revised: May $2021 \quad$ Accepted: May 2021)

Corresponding author: Chandana G. Email: chandanaggajendran@gmail.com

\begin{abstract}
Introduction and Aim: Non-alcoholic fatty liver disease (NAFLD) is one of the common causes of liver cirrhosis among general population. Studies have identified NAFLD and evaluated lipid profile in pregnant women. Some have explored association that existed between lipid levels and adverse pregnancy outcome in western countries. Our aim was to find the prevalence of non-alcoholic fatty liver disease (NAFLD) in pregnant women among rural population in their III trimester and to assess dyslipidemia in these women.
\end{abstract}

Materials and Methods: This is a prospective study conducted on 101 rural pregnant women coming for antenatal visits in their third trimester to Dept., of Obstetrics and Gynaecology, PESIMSR, Kuppam. Liver scan was done during the regular obstetric scan to identify NAFLD. All pregnant women underwent lipid profile testing (Triglyceride, Total Cholesterol, HDL, LDL, VLDL).

Results: There was a $1 \%$ prevalence of NAFLD among 101 rural expectant mothers. Lipid profile significantly $(\mathrm{p}<0.05)$ increased with increased BMI, parity and highly educated and fairly good socioeconomic background. Women with higher body mass index and triglyceride levels delivered babies with large birth weight $(\mathrm{p}<0.05)$.

Conclusion: There are metabolic derangements in pregnant women leading to dyslipidemia even among those who did not have NAFLD. High parity women and those with high atherogenicity are at cardiovascular risk diseases in future. We suggest further studies to establish biological reference ranges for lipid profile during pregnancy. There is a need for, pregnant women to be assessed for dyslipidemia and screened for NAFLD by scanning liver along with routine obstetric scan in tertiary care centre.

Keywords: NAFLD; dyslipidaemia; cirrhosis; lipid profile.

\section{INTRODUCTION}

$\mathrm{N}$ on-alcoholic fatty liver disease (NAFLD) is a condition whereby there is excessive accumulation of triglycerides in the liver without excess alcohol consumption. India being the capital for diabetes mellitus in the world, the prevalence of NAFLD among general population is high with prevalence of $15-40 \%$ in western countries and $9-32 \%$ in India (1). Liver being a visceral organ with distinct porto-venous circulation releases free fatty acids directly into the portal venous system unlike subcutaneous, adipose tissue which releases in the general circulation (2). Therefore, NAFLD causes elevated circulating lipid levels thereby leading to dyslipidaemia and metabolic syndrome.

Liver diseases that occur commonly in pregnancy like pre-eclampsia, obstetric cholestasis, acute fatty liver of pregnancy, HELLP (haemolysis elevated liver enzymes low platelet) syndrome and hyperemesis gravidarum are the major causes of maternal mortality and morbidity, thereby liver function tests are frequently done. However, both dyslipidaemia and NAFLD are overlooked during pregnancy.
With the literature search, most of the studies (3$8,9,10)$ have focused on the presence of NAFLD and evaluating lipid profile during the course of pregnancy from I to III trimester. Large cohort studies (2,9-20) explored association that existed between lipid levels and adverse pregnancy outcome in western countries.

Amundsen et al., (18) reported that pregnant women normally show an increase in levels of triglycerides (TG) and total cholesterol (TC) as gestational age progresses. The placenta takes up, metabolizes both TG and TC and transports to the fetus in various forms for fetal development (12). However, several studies in the past have shown varying conclusions on effect of increased circulating TG and total cholesterol levels on adverse pregnancy outcomes like preterm birth (PTB) (13), pregnancy-induced hypertension (PIH) (14), preeclampsia (15), and large for gestation (LGA) (16). In contrary decreased total cholesterol levels during pregnancy is associated with adverse pregnancy outcomes like preterm birth and small for gestational age newborns (SGA)(17). 
Vrijkotte et al., (12) measured non-fasting maternal total cholesterol and triglyceride levels during early pregnancy and correlated them with perinatal outcomes like PIH, preeclampsia, preterm birth, SGA, LGA and child loss. They showed association of increased triglyceride levels with adverse pregnancy outcome. Total cholesterol was not associated with any of the outcome measures.

The pathophysiological reasons for the above findings were given by various studies and in fact, most reports mainly focused on maternal lipid profiles in later part of pregnancy especially in III trimester. These reports reasoned out that the circulating lipids undergo peroxidation causing oxidative stress by progressively producing free radicals and lipid peroxides exert a direct harmful effect on the endothelium of placental vasculature, inducing endothelial dysfunction (19). Endothelial dysfunction in placenta is reported to be associated with maternal complications and newborn's growth retardation.

NAFLD is often asymptomatic and is incidentally diagnosed by biochemical abnormalities and therefore has not gained much importance. With increasing awareness of NAFLD by various studies in general population, we believe that the diagnosis should be considered in pregnant women. However, in India, especially among rural population, similar studies are still to be explored. By doing this study, we can find the prevalence of NAFLD and dyslipidemia in pregnant women among rural population.

The objective of our study was to assess the prevalence of non-alcoholic fatty liver disease (NAFLD) in rural pregnant women in III trimester and to assess dyslipidemia in these pregnant women.

\section{MATERIALS AND METHODS}

It is an observational prospective study conducted on 101 pregnant women coming for antenatal visits in their third trimester to Dept of OBG, PES Institute of Medical Sciences and Research, Kuppam from June 2018 to July 2018, for a period of 2 months. After obtaining prior approval from the institutional research and ethical committee we had started the study.

\section{Inclusion Criteria}

All pregnant women age over 18 years attending antenatal visits in their III trimester with singleton pregnancy, to Obstetrics and Gynaecology (OBG) department were included in the study.

\section{Exclusion criteria}

Patients with established cardiovascular diseases, thyroid dysfunction, excessive alcohol or other drug abuse, current or recent psychiatric treatment (up to 4 months) during pregnancy. Diabetic pregnant women, pregnant women with presence of acute infectious pathology, specific infection and sexually transmitted infections, known liver abnormalities. Lipid altering medication (antiepileptic drugs, steroids, insulin and antidepressants, thyroid hormone replacement therapy), Twin pregnancy, women not willing to take part in the study.

\section{Method of collection of data}

200 pregnant women attended III trimester antenatal visit from June 2018 to July 2018, out of which, based on our inclusion and exclusion criteria, around 101 pregnant women underwent liver scan for identifying NAFLD along with regular obstetric USG (ultrasonography) scan for fetal wellbeing. After taking informed consent these, 101 pregnant women with or without NAFLD underwent biochemical examination, where $5 \mathrm{ml}$ of venous blood in nonfasting state was drawn in a yellow gel vacutainer tube. Pregnant women were unwilling to come in fasting state due to peer and family pressure. Studies (27) reported, consuming average size breakfast caused minimal changes in lipid profile and also suggested non fasting triglyceride levels a better predictor of cardiovascular risk as compared to fasting triglyceride. Moreover studies (28) that used non fasting samples and fasting samples (16) reported results in accordance. The following tests were done in Vitros FS 5.1 and Vitros 250 autoanalyzers.

$>$ Triglyceride (Tgl): L- $\alpha$-glycerol-phosphate oxidase and peroxidase method

$>$ Total Cholesterol (TC): Cholesterol oxidase peroxidase method

$>$ High Density lipoprotein (HDL): Enzymatic method

$>$ Low density lipoprotein (LDL) and very lowdensity lipoprotein (VLDL): Calculated method using Freidman's calculation. $\mathrm{VLDL}=\mathrm{Tgl} / 5$ and $\mathrm{LDL}=\mathrm{TC}-(\mathrm{HDL}+\mathrm{VLDL})$. This formula cannot be used if Tgl levels are $\geq 450 \mathrm{mg} / \mathrm{dl}$.

IBM SPSS Statistical software version 15 was used for statistical analysis. Descriptive statistics were used to profile the sample by using ANOVA for continuous variables. Pearson coefficient of correlation to quantify the association between variables. In all analyses, a $P$ value 0.05 was considered statistically significant.

\section{RESULTS}

The pregnant women in the present study were from rural population. As per our inclusion and exclusion criteria, 101 pregnant women were screened for NAFLD using USG scan and tested along with lipid levels. Out of 101 pregnant women screened, prevalence of NAFLD was found to be $1 \%$ in our study (Graph 1).

BMI was classified according to WHO Classification. The mean BMI in women in their III trimester of 
pregnancy was $23.8 \mathrm{~kg} / \mathrm{m}^{2}$. Since most of the patients were from low socio-economic status only $10 \%$ of pregnant women were on the on obese side. Half of the study population (48\%) were found to have normal BMI with mean age of 23.3 years. All the lipid levels significantly increased as the body mass index (BMI) of the pregnant women increased with a $\mathrm{p}$ value of $<0.05$ (Table 1 ).

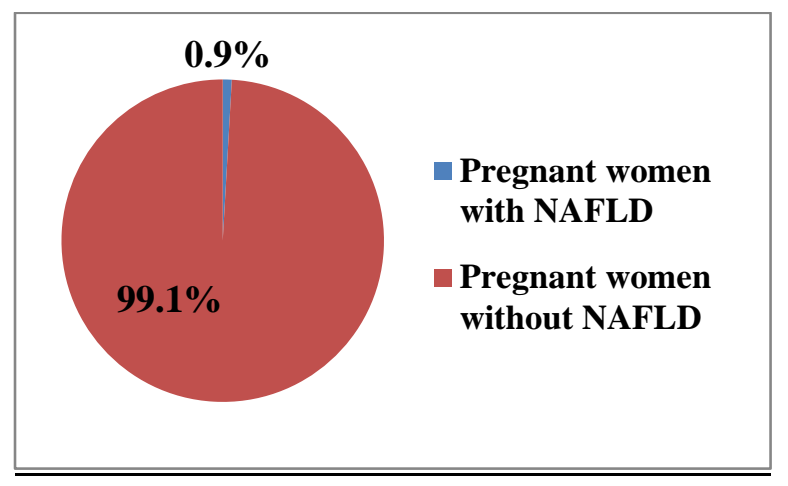

Graph 1: Prevalence of NAFLD in rural pregnant women

Table 1: BMI and Lipid levels of pregnant women

\begin{tabular}{|c|c|c|c|c|c|}
\hline $\begin{array}{c}\text { Parameter Total } \\
(n=100)\end{array}$ & $\begin{array}{c}<18.5(\mathrm{n}=11) \\
\left(\mathrm{kg} / \mathrm{m}^{2}\right)\end{array}$ & $\begin{array}{c}18.5-24.9(\mathrm{n}=48) \\
\left(\mathrm{kg} / \mathrm{m}^{2}\right)\end{array}$ & $\begin{array}{c}25-29.9(\mathrm{n}=31) \\
\left(\mathrm{kg} / \mathrm{m}^{2}\right)\end{array}$ & $\begin{array}{c}>30(n=10) \\
\left(\mathrm{kg} / \mathrm{m}^{2}\right)\end{array}$ & P value \\
\hline Triglyceride $(\mathrm{mg} / \mathrm{dl})$ & $85.27 \pm 45.95$ & $114.60 \pm 48.56$ & $154.26 \pm 63.36$ & $221.50 \pm 76.075$ & 0.000 \\
\hline Cholesterol (mg/dl) & $77.63 \pm 57.64$ & $102.25 \pm 47.80$ & $130.06 \pm 47.803$ & $151.30 \pm 27.24$ & 0.001 \\
\hline $\mathrm{HDL}(\mathrm{mg} / \mathrm{dl})$ & $22.58 \pm 10.53$ & $24.17 \pm 13.11$ & $31 \pm 10.90$ & $36.30 \pm 10.91$ & 0.005 \\
\hline VLDL $(\mathrm{mg} / \mathrm{dl})$ & $17.05 \pm 9.19$ & $22.92 \pm 9.71$ & $30.67 \pm 12.67$ & $38.3 \pm 19.54$ & 0.000 \\
\hline LDL (mg/dl) & $38.12 \pm 43.24$ & $55.17 \pm 33.85$ & $68.21 \pm 34.87$ & $76.70 \pm 20.25$ & 0.027 \\
\hline
\end{tabular}

Table 2: BMI and lipid levels according to parity of pregnant women

\begin{tabular}{|c|c|c|c|c|}
\hline Parity Total $(\mathbf{n}=\mathbf{1 0 0})$ & $\mathbf{0}(\mathbf{n}=\mathbf{1 7})$ & $\mathbf{1}(\mathbf{n}=\mathbf{5 0})$ & $\geq \mathbf{2}(\mathbf{n}=\mathbf{3 3})$ & P value \\
\hline BMI & $20.18 \pm 3.14$ & $24.6 \pm 4.29$ & $24.51 \pm 4.80$ & 0.002 \\
\hline Triglyceride $(\mathrm{mg} / \mathrm{dl})$ & $75.06 \pm 31.23$ & $143.31 \pm 63.87$ & $151.39 \pm 68.23$ & 0.000 \\
\hline Cholesterol $(\mathrm{mg} / \mathrm{dl})$ & $81.35 \pm 39.84$ & $111.31 \pm 46.40$ & $131.76 \pm 55.68$ & 0.003 \\
\hline HDL $(\mathrm{mg} / \mathrm{dl})$ & $21.24 \pm 11.18$ & $27.22 \pm 12.24$ & $30.155 \pm 13.15$ & 0.045 \\
\hline VLDL $(\mathrm{mg} / \mathrm{dl})$ & $15.00 \pm 6.2$ & $28.66 \pm 12.77$ & $28.46 \pm 13.56$ & 0.000 \\
\hline LDL $(\mathrm{mg} / \mathrm{dl})$ & $45.14 \pm 25.53$ & $55.64 \pm 33.04$ & $73.47 \pm 37.71$ & 0.017 \\
\hline
\end{tabular}

The lipid levels did vary significantly with $\mathrm{p}$ value of $<0.05$ in accordance with parity, whereby the triglyceride levels, total cholesterol, LDL levels were higher in multiparous women than the nulliparous women (Table 2)

Table 3: Education level and lipid levels of pregnant women

\begin{tabular}{|c|c|c|c|c|}
\hline Age Total $(\mathbf{n}=\mathbf{1 0 0})$ & $\mathbf{\leq 1 0}^{\text {th }} \mathbf{s t d}(\mathbf{n}=\mathbf{5 5})$ & $\mathbf{1 1 - 1 2}$ std $(\mathbf{n = 2 7})$ & Degree $(\mathbf{n = 1 8})$ & P value \\
\hline Triglyceride $(\mathrm{mg} / \mathrm{dl})$ & $121.62 \pm 58.11$ & $137.26 \pm 52.34$ & $168.94 \pm 95.08$ & 0.03 \\
\hline Cholesterol $(\mathrm{mg} / \mathrm{dl})$ & $105.75 \pm 51.49$ & $118.00 \pm 49.4$ & $128.06 \pm 51.07$ & 0.23 \\
\hline HDL $(\mathrm{mg} / \mathrm{dl})$ & $25.53 \pm 12.59$ & $29.52 \pm 13.65$ & $29.44 \pm 10.99$ & 0.30 \\
\hline VLDL $(\mathrm{mg} / \mathrm{dl})$ & $23.23 \pm 11.01$ & $27.15 \pm 10.46$ & $33.7 \pm 19.01$ & 0.01 \\
\hline LDL $(\mathrm{mg} / \mathrm{dl})$ & $56.99 \pm 35.81$ & $61.029 \pm 36.68$ & $64.8 \pm 33.13$ & 0.69 \\
\hline
\end{tabular}

Most of the study subjects were from rural population, almost half of study participants $(55 \%)$ were educated up to $10^{\text {th }}$ standard, only $18 \%$ were graduates and among the graduates, only $6 \%$ were employed with sedentary work style. $10 \%$ of them were agriculturist, and other women were found to be homemakers. Our study showed that triglyceride and VLDL levels in graduate women were significantly high, whereas other lipid levels did not vary significantly (Table 3 ).

Table 4: Fetal outcome (Birth weight) as compared to the BMI $(\mathrm{kg} / \mathrm{m} 2)$ of pregnant women $(\mathrm{n}=35)$

\begin{tabular}{|c|c|c|c|c|c|}
\hline $\begin{array}{c}\text { Parameter BMI } \\
\text { (Total: } \mathbf{n = 3 5})\end{array}$ & $\begin{array}{c}<\mathbf{1 8 . 5}(\mathbf{k g} / \mathbf{m} 2) \\
(\mathbf{n}=\mathbf{2})\end{array}$ & $\begin{array}{c}\mathbf{1 8 . 5}-\mathbf{2 4 . 9} \\
(\mathbf{k g} / \mathbf{m} 2)(\mathbf{n}=\mathbf{2 0})\end{array}$ & $\begin{array}{c}\mathbf{2 5}-\mathbf{2 9 . 9}(\mathbf{k g} / \mathbf{m} 2) \\
(\mathbf{n}=\mathbf{9})\end{array}$ & $\begin{array}{c}\mathbf{3 0}(\mathbf{k g} / \mathbf{m} 2) \\
(\mathbf{n}=\mathbf{4})\end{array}$ & $\mathbf{P}$-value \\
\hline Birth weight & $2.25 \pm 1.06$ & $2.74 \pm 0.56$ & $3.05 \pm 0.29$ & $3.41 \pm 0.28$ & 0.035 \\
\hline
\end{tabular}

35 of 101 pregnant women could be followed up till delivery and birth weight of the newborns were incorporated in the study, however other 66 birth weights could not be included, as either these women with gestational weeks of $28-31$ weeks did not deliver before the study was completed or lost to follow up. Babies born to mothers with high BMI were found to have higher birth weight compared to mothers with low BMI. There was 2 newborn deaths in mothers with BMI of $<18.5 \mathrm{~kg} / \mathrm{m}^{2}$, one of death was due to preterm birth (Table 4). 


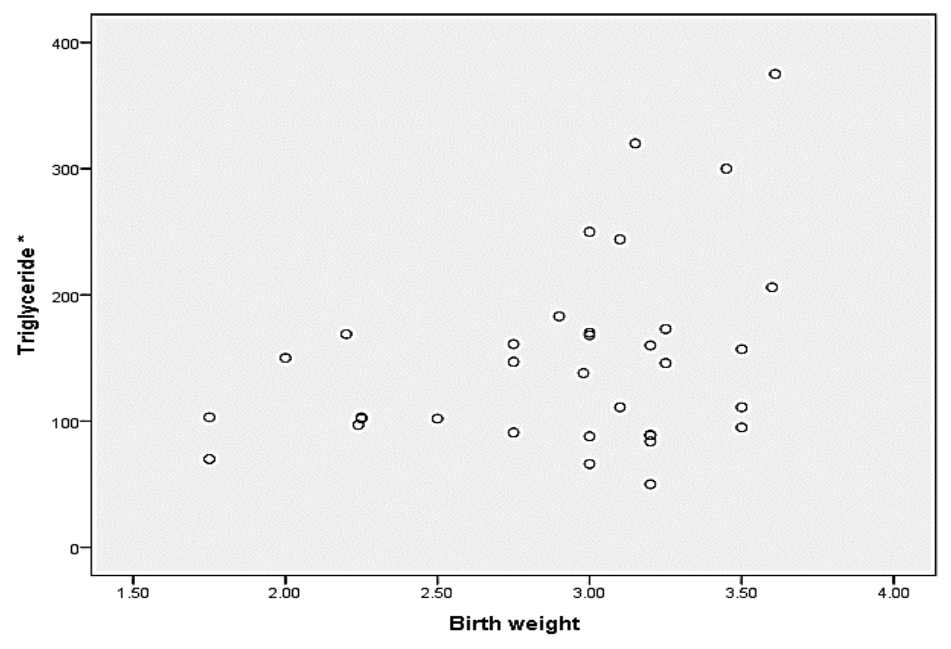

Graph 2: Correlation between fetal outcome (birth weight) and triglyceride levels of pregnant women.

The mean birth weight of the babies born from our study population was $2.3 \mathrm{kgs}$. The birth weight was found to be high in women with high triglyceride levels with $r=0.334$ and $p$ value:0.05 (Graph 2).

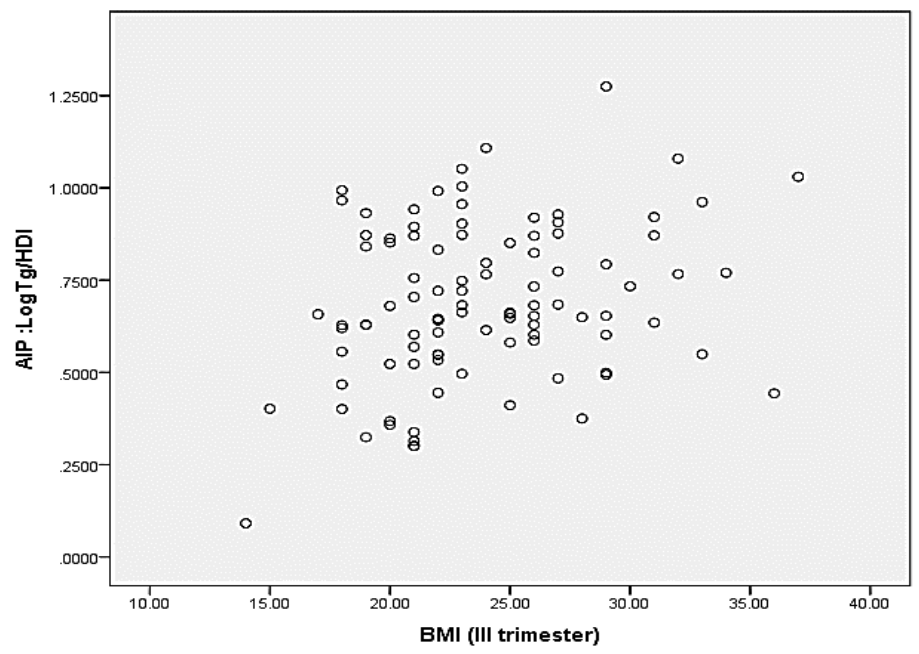

Graph 3: Correlation between Atherogenic index of plasma with BMI of pregnant women.

There is weak positive correlation between atherogenic index of plasma Log (Tg/HDL) and BMI of pregnant women with $\mathrm{r}=0.271$ and $\mathrm{p}$ value 0.007(Graph 3).

\section{DISCUSSION}

Freinkel et al., et al (21) was first to describe altered metabolic functioning in late pregnancy as "accelerated starvation" when food is unavailable and "facilitated anabolism" when food is ingested. In western population in Hagestorm et al., (9) found a prevalence of NAFLD of $0.005 \%$ in Swedish pregnant women, the study done from 1999- 2011, but till date in Indian population there is no evidenced prevalence of NALFD in pregnant women. We found $1 \%$ prevalence of NAFLD in our study. Studies like De Souza et al., (10) have showed that sonographic assessment of NAFLD has limited ability to detect small amount of hepatic steatosis and to distinguish various stages of NAFLD. Hence, they recommended MRI for screening NAFLD which was again not cost effective in our population. Since the sample size of NAFLD was very low as compared to the sample size of pregnant women without NAFLD, we could not correlate the lipid levels between these groups. However, we could derive to a conclusion that there was an existence of lipid derangements in pregnant women even without NAFLD. Mean gestational age of pregnant women taken into our study was 33 weeks. The mean triglyceride, total cholesterol, HDL, VLDL and LDL, in our population is $134.36 \pm 66.5, \quad 113.07 \pm 51.135, \quad 27.31 \pm 12.65$, $26.27 \pm 13.14$, and $59.7 \pm 34.85$ respectively. But in Pusukuru et al., (3) a study population done in Mumbai (Urban population) the mean triglyceride, cholesterol, HDL, LDL and VLDL levels in third trimester were $216.78 \pm 20.09 \mathrm{mg} / \mathrm{dl}, 242.65 \pm 20.44$ $\mathrm{mg} / \mathrm{dl}, 43.07 \pm 4.34 \mathrm{mg} / \mathrm{dl}, 137.82 \pm 13.45 \mathrm{mg} / \mathrm{dl}$ and $36.27 \pm 6.72 \mathrm{mg}$ respectively. These values were in accordance with many other studies $(2,12)$ among western population. The serum cholesterol levels are determined by endogenous synthesis and dietary intake. Acetyl CoA is the main substrate for synthesis of cholesterol in liver. Acetyl CoA is produced by oxidation of fatty acid which is dependent on exogenous and endogenous synthesis of triglycerides 
(22). All women in our study population came from a rural background with low socioeconomic status, their nutrition is at stake therefore pregnant women in our population have low cholesterol levels and subsequently low lipoproteins. Anuradha et al., (23) study showed a mean of Tgl, Total cholesterol, HDL, LDL, and VLDL respectively 126.85 \pm 16.68 , $136.85 \pm 10.44,39 \pm 1.49,72.75 \pm 9.62$ and $24.7 \pm 3.05$ respectively. This study was likewise done among rural population in Ananthapuram in South India where women came from poor socioeconomic status similar to our study. Therefore, our lipid profile was closely associated with their study. Since there is no standard reference interval for lipid profile in pregnant women. This study expresses the need for intensive research on the importance of establishing biological reference ranges for lipid profile levels, in pregnant women at different trimesters. Ethnicity, diet, socioeconomic background, occupation, pre pregnancy weight and other habits needs consideration for establishing reference ranges, hence reference ranges established for individual population seems favourable. During pregnancy there is increase in maternal blood volume and therefore the maternal body metabolism shifts to utilization of fat from that of carbohydrate. Increase in insulin resistance and plasma lipolytic hormonal concentration is an additional reason for altered metabolism. The rise in triglycerides is due to two reasons (3)

a. White adipose tissue acts like an endocrine organ which releases leptin, adiponectin and other paracrine factors termed adipokines. There is synergistic activity of both hepatic lipase and lipoprotein activity, leading to enhanced hepatic triglyceride synthesis and decreased catabolism of adipose tissue.

b. Apolipoproteins A-I, A-II, and B also rise across gestation. HDL-cholesterol concentrations initially increase and then fall in the third trimester therefore HDL is lower side in later part of pregnancy.

The mean age of study participants was 23.3 yrs. The lipid levels did not significantly increase with age. However, half of them were falling into normal BMI. Maternal BMI also had influence on lipid levels, where women with higher BMI had significantly high levels of lipids. The findings of our study were similar to the Geraghty et al., (2), Vatrain et al., (24) and Sceifres et al., (25) which also showed significant differences in blood Triglyceride level in women with overweight and later period of pregnancy. Our study showed significant positive correlation between total cholesterol levels and BMI whereas different other studies (2, 30-31) were not statistically significant. This difference observed in lipid levels between BMI categories could be due to the influence of insulin resistance in overweight and obese women. All the lipid levels considerably increased with multiparous women which is in accordance with Akter et al., (26) and Vrijkotte et al., (12) which showed significantly high lipid levels with increase in parity. However, Akter et al., (26) did the study in rural Bangladeshi non pregnant women and Vrijkotte et al., (12) during early pregnancy. Both reported multiparity and gravidity a risk factor for metabolic syndrome. In our population none of the women where smokers nor were alcoholic. Out of 101 women included in the study majority of the women were housewives and were moderate workers. 7 women were agriculturist doing heavy work and 6 women were employed in sedentary work environment. Women working in sedentary work environment had significantly high triglyceride levels.

Among our study population pregnant women in III trimester with high triglyceride levels especially in obese category delivered babies with high birth weight, however they were not macrosomic or LGA babies as reported by Geraghty et al (2). Underweight pregnant women with low cholesterol and low triglyceride levels delivered low birth weight babies. One woman out of 35 pregnant women who delivered had preeclampsia at the time of delivery and rest of the women pregnancy and deliveries were uneventful. The pathophysiology behind pregnant women with high triglyceride delivering babies with large for gestational age (LGA) is, fatty acids which are produced by higher triglycerides could act as growth factors and high levels of free fatty acids are known to compete for hormones bound to albumin (e.g., sex hormones). This leads to subsequent increase in free hormone levels, thereby assisting in placenta capacity, intrauterine growth and development (12).

Studies in the past suggest that pregnant women with high atherogenic index of plasma were prone for increased risk for preeclampsia (maternal outcome) and risk for cardiovascular diseases (26). Our study showed significant $(\mathrm{p}<0.05)$ positive correlation of BMI and atherogenic index of plasma. Out of 35 pregnant women followed up till delivery. 2 ladies were obese with high atherogenic index; however, their maternity outcome was uneventful. These women might be at risk for cardiovascular diseases in future.

At the outset, we have few limitations in this study. The study period was of short duration for a period of 2 months. In these two months we got $1 \%$ prevalence of NAFLD, however if we continue our study for a longer duration, we would have got sufficient number of cases for comparing lipid levels in pregnant women with NAFLD and pregnant women without NAFLD. Moreover, screening of NAFLD and comparison of lipid profiles in pre pregnancy, in all the trimesters of pregnancy and post pregnancy with larger sample size is desirable, but not financially 
feasible. Majority of pregnant women in rural population have antenatal visits in later pregnancy hence we could recruit pregnant women in III trimester.

\section{CONCLUSION}

We found only $1 \%$ prevalence of NAFLD for study period of 2 months. Hence, we could not get sufficient sample size of NAFLD to we could not correlate the lipid levels between these pregnant women with NAFLD and pregnant women without NAFLD. However, we could derive to a conclusion that there was an existence of lipid derangements in pregnant women even without NAFLD. Many western studies and Indian studies among urban population studies have reported dyslipidemia in early and late pregnancy. Few of these studies have concluded that lipid profile should be a part of a routine investigation during antenatal check up. To the best of our knowledge this is the only study that focused on the prevalence of NAFLD in rural pregnant women. We would like to conclude that due to rapid urbanization of the rural parts of our country, there is a need for pregnant women to be assessed for dyslipidemia. Lipid profile testing and scanning liver along with routine obstetric scan can be done in tertiary care centres where facilities are available, but in rural pregnant expectant mothers where facilities are not available in primary health centres, we can assess dyslipidemia by doing lipid profile. This study also shows the importance of strategic efforts to avoid maternal and fetal adverse outcomes as well as to focus on life style modification and nutrition to control metabolic derangements both during and after pregnancy. In addition, we suggest further research necessary to establish biological reference ranges for lipid profile during pregnancy.

\section{ACKNOWLEDGMENT}

The project was selected and approved by ICMR STS-2018. I sincerely thank the ICMR for encouraging students and faculty members in research projects.

\section{CONFLICT OF INTEREST}

Authors declare no conflict of interest.

\section{REFERENCES}

1. Prabhakar, N., Ambali, N. R., Unnikrishnan, T. K., Renymol, B. Prevalence of non-alcoholic fatty liver disease (NAFLD) in patients with type 2 diabetes mellitus and its correlation with coronary artery disease (CAD). International Journal of Research in Medical Sciences. 2017; 5(12): 5175-5181.

2. Foghsgaard, Sandreasen, C., Vedtofte, L., Anderson, E.S., Bahne, E., Strandverg C., et al., Non-alcoholic fatty liver disease is prevalent in women with prior gestational diabetes mellitus and independently associated with insulin resistance and waist circumference. Diabetes Care. 2017; 40(1): 109-116.
3. Geraghty, A. A., Alberdi, G., Sullivan, E. J., McAuliffe, M. F. Maternal and fetal blood lipid concentrations during pregnancy differ by maternal body mass index: finding from ROLO study.BMC pregnancy and childbirth 2017: 360

4. Pusukuru, R., Shenoi, A. S., Kyada, P. K., Ghodke, B., Mehta, V., Bhuta, K., Bhatia, A. Evaluation of Lipid Profile in Second and Third Trimester of Pregnancy. Journal of Clinical and Diagnostic Research. 2016; 10(3): QC12QC16.

5. Lippi, G., Albiero, A., Montagnana, M., Salvagno, G. L., Scevarolli, S., Farnchi, M., et al., Lipid and Lipoprotein profile in physiological pregnancy. Clin Lab. 2007; 53: 173177.

6. Jamil, A. A. T., Elsoni, B., Zaki, H. Y., Elbadawi, N. E., Ahmed, E. G., Ibrahim, E. K., et al. Assessment of lipid profile in sudanese pregnant women. Key Research Journal of Biotechnology. 2013; 1(1): 4-15.

7. Okojie, F. O., Idonije, O. B., Eseigbe, M. A., Okhiai, O., Unuabonah, F., Dike, M., et al. Comparative study of lipid profile of normal pregnant women in the different trimesters. Archives of Applied Science Research. 2011; 3(3): 528-532.

8. Phuse, S. S., Effective study of lipid profile during pregnancy. International Journal of Applied Biotechnology and Biochemistry. 2012; 2(4): 381-386.

9. Hagstrom, H., Hoijer, J., Ludvigsson, J. F., Bottai, M., Ekbom, A., Hultcrantz, R., et al. Adverse outcomes of pregnancy in women with non alcoholic fatty liver disease. Liver International.2016; 36(2): 268-274.

10. Souza, L. R. D., Berger, H., Vlachou, H. R. P. V., Maguire, J. L. Non-alcoholic fatty liver disease in early pregnancy predicts dysglycemia in mid-pregnancy: prospective study. The American Journal of Gastroenterology. 2016; 111: 665-670.

11. Castracane, V., Davis, G., Martinez, B., Maguire, C., Kauffman, R. Non-alcoholic fatty liver disease in pregnant women. Non-alcoholic fatty liver disease in pregnant women. Endocrine Abstracts. 2012; 29: P938

12. Vrijkotte, T. G. M., Krukziener, N. T., Hutten, C. K., Eijsden, V. M. V., Twickler, M. B. Maternal Lipid Profile During Early Pregnancy and Pregnancy Complications and Outcomes: The ABCD Study. J Clin Endocrinol Metab. November. 2012; 97(11): 3917-3925.

13. Catov, J. M, Ness, R. B., Wellons, M. F., Jacobs, D. R., Roberts, J. M., Gunderson, E. P. Pre-pregnancy lipids related to preterm birth risk: the coronary artery risk development in young adults study. J Clin Endocrinol Metab. 2010; 95: 3711-3718.

14. Jan, M. R., Nazli, R., Shah, J., Akhtar, T. A study of lipoproteins in normal and pregnancy induced hypertensive women in tertiary care hospitals of the North West Frontier Province-Pakistan. Hypertens Pregnancy. 2012; 31: 292299.

15. Ziaei, S., Bonab, K. M., Kazemnejad, A. Serum lipid levels at 28-32 weeks' gestation and hypertensive disorders. Hypertens Pregnancy. 2006; 25: 3-10.

16. Di Cianni, G., Miccoli, R., Volpe, L., Lencioni, C., Ghio, A., Giovannitti, M. G., et al., Maternal triglyceride levels and newborn weight in pregnant women with normal glucose tolerance. Diabet Med. 2005; 22: 21-25.

17. Edison, R. J., Berg, K., Remaley, A., Kelley, R., Rotimi, C., Stevenson, R. E., et al., Adverse birth outcome among mothers with low serum cholesterol. Pediatrics, 2007; 120: 723-733.

18. Amundsen, A. L., Khoury, J., Iversen, P. O., Bergei, C., Ose, L., Tonstad, S., et al., Marked changes in plasma lipids and lipoproteins during pregnancy in women with familial hypercholesterolemia. Atherosclerosis. 2006; 189: 451-457.

19. Hubel, C. A. Dyslipidemia, iron, and oxidative stress in preeclampsia: assessment of maternal and feto-placental interactions. Semin Reprod Endocrinol.1998; 16: 75-92.

20. Kaar, J. L., Crume, T., Brinton, J. T., Bischoff, K. J., McDuffie, R., Dabelea, D et al., Maternal obesity, 
gestational weight gain and offspring adiposity: the EPOCH Study. J Pediatr. 2014; 2165(3): 509-515.

21. Freinkel, N. Effect of the conceptus on maternal metabolism during pregnancy. Excerpta Medica. 1964; 12: 679-681.

22. Burtis, C. A. Lipids, lipoproteins, apolipoproteins and other cardiovascular risk factors. Tiez Text book of Clinical Chemistry and molecular diagnostics. $5^{\text {th }}$ edition 731-807.

23. Anuradha, R., Durga, T. Estimation of lipid profile among preeclampsia woman by comparing with normal pregnancy. International Journal of Contemporary Medical Research. 2016; 3(7): 1958-1961.

24. Vahratain, A., Misra, V. K., Trudeau, S., Misra, D. P. Prepregnancy body mass index and gestational age dependent changes in lipid levels during pregnancy. Obstretic Gynecol 2010; 116: 107-113.

25. Scifres, C. M., Catov, J. M., Simhan, N. H. The impact of maternal obesity and gestational weight gain on early and midpregnacy lipid profile. Obesity. 2014; 22: 932-938.

26. Akter, S., Jesmin, S., Rahman, M., Islam, M. M., Kathun, M. T., Yamaguchi, N., et al., Higher gravidity and parity are associated with increased prevalence of metabolic syndrome among rural Bangladeshi women. PLOS ONE. 2013; 8(8) e 68319.

27. Nigam, P. K. Serum lipid profile: fasting or non-fasting. Indian journal of clinical biochemistry. 2011; 26(1): 96-97.

28. Magnussen, E. B., Vatten, L. J., Lund-Nilsen, T. I., Salvesen, K. A., Davey Smith, G., Romundstad, P. R. Prepregnancy cardiovascular risk factors as predictors of preeclampsia: population-based cohort study. BMJ. 2007; 335: 978. 